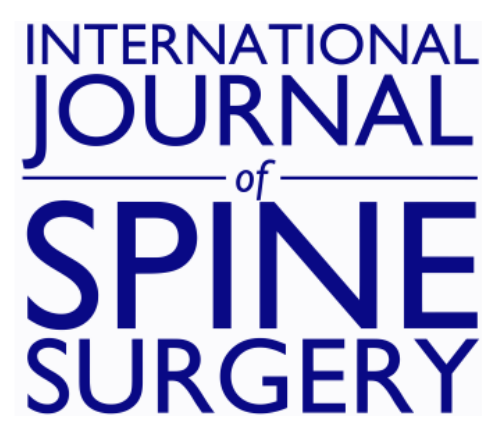

\title{
The Utility of Magnetic Resonance Imaging for Detecting Unstable Cervical Spine Injuries in the Neurologically Intact Traumatized Patient Following Negative Computed Tomography Imaging
}

JACOB FENNESSY, JOSEPH WICK, FIONA SCOTT, ROLANDO ROBERTO, YASHAR JAVIDAN and ERIC KLINEBERG

Int J Spine Surg 2020, 14 (6) 901-907

doi: https://doi.org/10.14444/7138

http://ijssurgery.com/content/14/6/901

This information is current as of April 26, 2023.

Email Alerts Receive free email-alerts when new articles cite this article. Sign up at: http://ijssurgery.com/alerts 


\title{
The Utility of Magnetic Resonance Imaging for Detecting Unstable Cervical Spine Injuries in the Neurologically Intact Traumatized Patient Following Negative Computed Tomography Imaging
}

\author{
JACOB FENNESSY, MD, JOSEPH WICK, MD, FIONA SCOTT, MD, MPH, ROLANDO ROBERTO, MD, \\ YASHAR JAVIDAN, MD, ERIC KLINEBERG, MD, MS \\ University of California, Davis, Sacramento, California
}

\begin{abstract}
Background: Neurologically intact blunt trauma patients with persistent neck pain and negative computed tomography (CT) imaging frequently undergo magnetic resonance imaging (MRI) for evaluation of occult cervical spine injury. There is a paucity of data to support or refute this practice. This study was therefore performed to evaluate the utility of cervical spine MRI in neurologically intact blunt trauma patients with negative CT imaging.

Methods: A retrospective review was performed of all neurologically intact blunt trauma patients presenting to a level 1 trauma center from 2005 to 2015 with persistent neck pain and negative CT imaging. The proportion of patients with positive MRI findings, subsequent treatment, and time required to obtain MRI results was evaluated.

Results: Of 223 patients meeting inclusion criteria, 11 had positive MRI findings; however, no patients were found to have unstable injuries requiring surgical treatment. The process for a complete evaluation of unstable cervical spine injury from the time of obtaining a CT scan was 19 hours and 43 minutes.

Conclusions: Eleven patients had positive MRI findings, yet these findings did not alter treatment. In contrast, the time required to obtain MRI results may substantially delay patient care.

Level of Evidence: IV (retrospective case series)

Clinical Relevance: Our results demonstrate that MRI has limited utility in neurologically intact blunt trauma patients with negative CT imaging.

Cervical Spine

Keywords: blunt trauma, cervical spine, magnetic resonance imaging, computed tomography, occult injury, posterior elements, unstable, ligamentous
\end{abstract}

\section{BACKGROUND}

Detection of cervical spine injury among neurologically intact blunt trauma patients with persistent neck pain and negative computed tomography (CT) imaging presents an important clinical challenge. The overall rate of cervical spine injury in blunt trauma patients is $7 \%-15 \%,{ }^{1}$ and the NEXUS criteria are widely used to guide clinical evaluation of cervical spine injury. ${ }^{2}$ CT scan is the recommended imaging modality, ${ }^{3}$ as it is highly sensitive for bony injury. ${ }^{2}$ However, CT's ability to adequately detect isolated, unstable ligamentous cervical spine injury in blunt trauma patients is controversial. This is especially true given the potentially devastating consequences of a missed unstable injury. ${ }^{4,5}$ Clinicians are often concerned that persistent neck pain in the setting of negative CT may represent occult, unstable ligamentous injury. Therefore, many centers utilize magnetic resonance imaging (MRI) to evaluate for injury to the cervical spine discoligamentous complex. However, this too is controversial, as MRI has a high false-positive rate, potentially leading to unnecessary cervical collar treatment as well as delay of care while MRI is obtained. ${ }^{1,2,4,6}$ To date, studies assessing the efficacy of MRI in blunt trauma patients with negative $\mathrm{CT}$ and persistent neck pain have yielded mixed results; ${ }^{7-15}$ a recent metaanalysis concluded that insufficient data exist for determining whether MRI should be used in this population. ${ }^{4}$ Given the paucity of useful data, we performed a retrospective review of neurologically intact blunt trauma patients presenting to our level 
1 trauma center with persistent neck pain and negative CT to assess whether MRI affected treatment course. As secondary outcomes, we aimed to assess whether MRI delayed care and whether injury mechanism affected MRI utility. We hypothesized that MRI would not affect treatment, regardless of mechanism, and that time required to obtain MRI may substantially delay patient care.

\section{METHODS}

\section{Patient Population and Inclusion/Exclusion Criteria}

Institutional review board approval was obtained for this study. All patients age $>18$ presenting to our level 1 trauma center with axial cervical spine pain or tenderness following blunt trauma over the 10-year period from January 1, 2005, to January 1, 2015, who underwent both CT and MRI were eligible for inclusion. Current Procedural Terminology (CPT) codes for cervical spine CT (72125, 72126, 72127) and MRI (72141, 72142, 72156) were used to identify patients who underwent both imaging types. Patients with unreliable exams or who were uncooperative due to intoxication or altered mental status were included if documented to be grossly moving all extremities, and there was no documentation of objective focal neurological deficit. Patients were excluded for penetrating trauma or if CT demonstrated cervical spine fracture, subluxation, or dislocation. Patients were also excluded for prior cervical spine surgery, presence of instrumentation, or neurologic deficits on exam. Figure 1 demonstrates patient enrollment.

\section{Imaging Acquisition and Interpretation}

CT examinations were performed using 1 of $2 \mathrm{GE}$ Light Speed VCT 64-slice scanners with 70-cm bore size and 500-1b weight limit. MRI examinations were performed using 1 of 3 GE Signa-HDX 1.5 Tesla or 3.0 Tesla scanners with $50-\mathrm{cm}$ bore size and 300-lb weight limit. MR imaging series included T1and T2-weighted sagittal, axial, and coronal plane imaging as well as sagittal Short-T1 Inversion Recovery series. All imaging was formally reviewed by attending faculty musculoskeletal and neuroradiologists within our institution; results of their interpretations were used in the analysis. MRI findings were considered to be positive if injuries were identified to any of the 3 columns as described by Denis. ${ }^{16}$

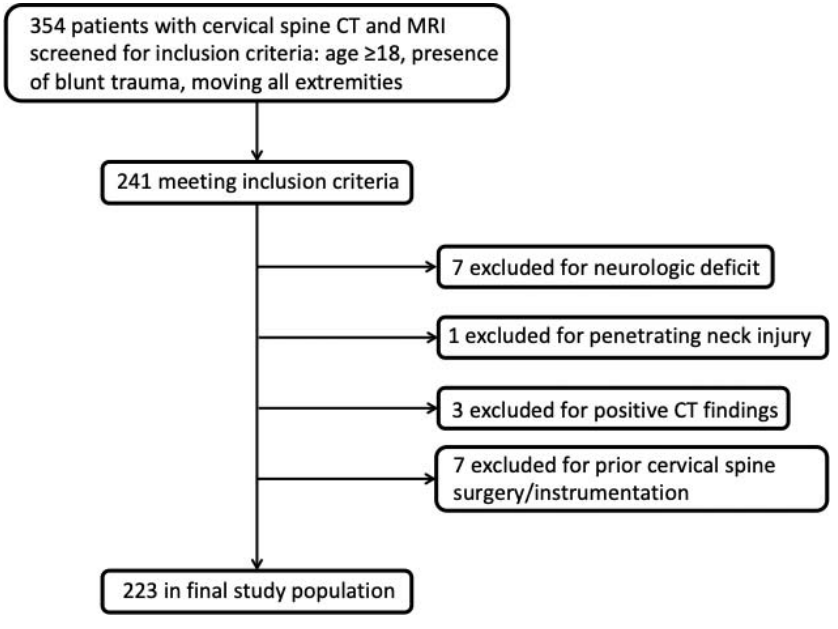

Figure 1. Patient enrollment with inclusion and exclusion criteria.

\section{Data Collection and Covariates}

For all included patients, age, gender, mechanism of injury, treatment/intervention, disposition, and indication for MRI were abstracted from the electronic medical record. Injury mechanisms were classified as high or low energy based on the American College of Surgeons National Surgical Quality Improvement Program trauma criteria. ${ }^{17,18}$ Timing of CT and MRI were determined by evaluating electronic time stamps on emergency department (ED) admission documentation, CT and MRI time stamps, and radiology reports.

\section{Statistical Methods}

Descriptive statistics, including mean and standard deviation, median and interquartile range, and percent, were calculated for patient covariates (Table 1). The difference in prevalence of positive MRI findings between patients with high- and lowenergy mechanisms was assessed using the Fisher exact test. Times from CT study to MRI study, MRI study to attending radiologist MRI read, and CT study to attending radiologist MRI read and total time of hospitalization were calculated for each patient. Time from CT study to attending radiologist MRI read was calculated as a surrogate for the entire time elapsed in a complete evaluation of patients for cervical spine injury. Median values and interquartile ranges were calculated for each time category to reduce the effect of outlier values. A $P$ value $<.05$ was considered statistically significant. 
Table 1. Patient demographics, magnetic resonance imaging (MRI) results, and treatment for all patients meeting the inclusion criteria $(n=223)$. "Other low energy" includes history of patient found down without recollection of incident and without evidence of high-energy trauma. "Other high energy" includes snowboarding or contact sporting activities and so on. Fall from height is defined as a height taller than a bed per the American College of Surgeons National Surgical Quality Improvement Program trauma criteria. ${ }^{17,18}$

\begin{tabular}{lcc}
\hline Variable & Number & $\begin{array}{c}\text { Standard } \\
\text { Deviation (\%) }\end{array}$ \\
\hline Total patients & 223 & Not applicable \\
Age (y) & 43.9 & 19.3 \\
Male gender & 115 & 51.6 \\
Female gender & 108 & 48.4 \\
Mechanism of injury & & \\
High-energy mechanism & 172 & 77.1 \\
$\quad$ Motor vehicle collision & 102 & 45.7 \\
$\quad$ Fall from height & 22 & 9.9 \\
$\quad$ Assault & 26 & 11.7 \\
Auto versus pedestrian & 9 & 4.0 \\
Other high energy & 9 & 4.0 \\
Low-energy mechanism & 40 & 17.9 \\
$\quad$ Ground-level fall & 33 & 3.1 \\
Other low energy & 7 & 6.7 \\
$\quad$ Unknown & 15 & 4.5 \\
Positive MRI findings & & 0.4 \\
Interspinous ligament signal change & 10 & \\
Prevertebral edema & 1 & 81.8 \\
Treatment of patients with positive & & 18.2 \\
$\quad$ MRI findings & 9 & 0 \\
Cervical collar & 2 & \\
No immobilization & 0 & \\
Surgery & & \\
\hline
\end{tabular}

\section{RESULTS}

A total of 354 patients were evaluated for study inclusion; 223 were included in the final study population. Table 1 shows demographic data, mechanism of injury, and MRI findings with subsequent treatment for patients with positive MRI findings. A total of $115(51.6 \%)$ of patients were male, with a mean age of 44 years. Seventyseven percent of patients had a high-energy mechanism, with $102(45.7 \%)$ being involved in a motor vehicle collision. Of all patients, $11(4.9 \%)$ had positive MRI findings defined as abnormalities or signal changes within 1 or more of the 3 structural columns, including 10 with posterior interspinous ligament edema and 1 with anterior cervical spine soft tissue (prevertebral) edema (Figures 2 and 3). No additional fractures or evidence of instability were identified on MRI. Nine of the 11 patients with positive MRI findings were treated with either soft or rigid cervical orthoses. No patients were treated surgically. Table 2 shows demographics, injury mechanism and characteristics, and outcomes for the patients with positive MRI findings. No significant difference in rate of MRI-identified injury was found between patients with a highversus low-energy mechanism of injury $(P<.05)$.

For all patients, the median time elapsed between obtaining an MRI study and attending radiologist MRI read was 10 hours and 27 minutes, with the median time between initial CT study and attending radiologist MRI read being 19 hours and 43 minutes. Table 3 summarizes the time required to obtain MRI studies, time to attending radiologist MRI read, and total hospitalization time.

\section{DISCUSSION}

Blunt trauma patients who have negative cervical spine CT imaging but persistent neck pain present a challenging clinical scenario. In an effort to rule out
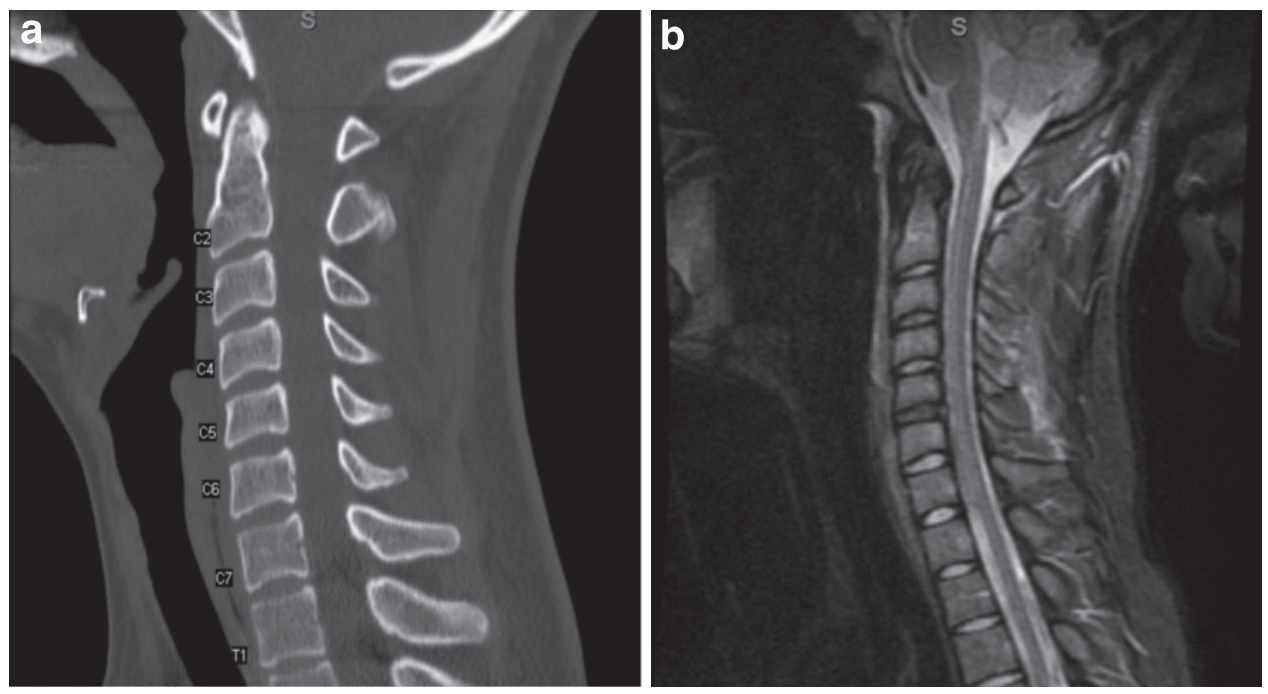

Figure 2. Twenty-five-year-old female involved in a motor vehicle collision complaining of midline cervical tenderness and painful range of motion on exam following an unremarkable cervical spine computed tomography scan (left). Magnetic resonance imaging was obtained to rule out unstable injury and noted to have interspinous ligament sprain from C3 to C6 demonstrated on Short-T1 Inversion Recovery imaging (right). 

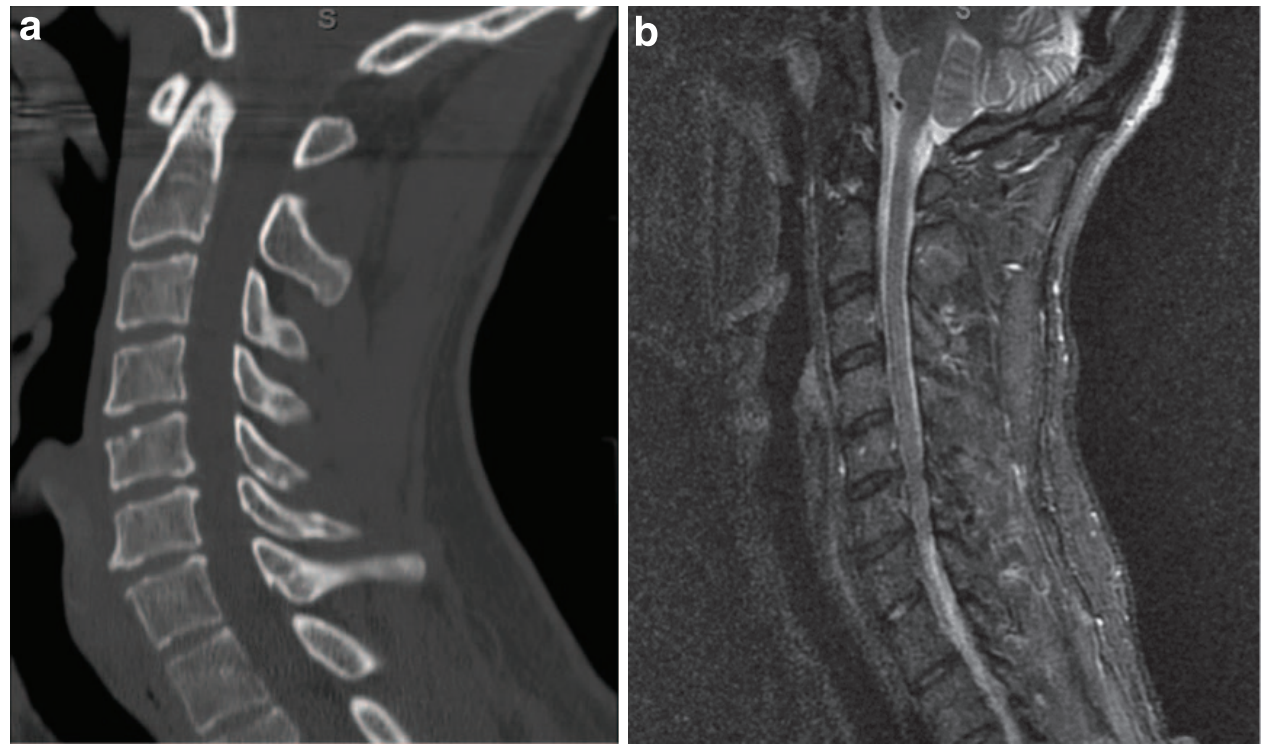

Figure 3. Thirty-seven-year-old male involved in auto-versus-pedestrian accident complaining of midline cervical tenderness and painful range of motion (ROM) on exam. Cervical spine computed tomography is unremarkable for fracture or column disruption (left); however, magnetic resonance imaging was obtained and demonstrates prevertebral edema localized anterior to the C3/4, C4/5, and C5/6 vertebral bodies (right).

missed, unstable cervical spine injuries in these patients, MRI is frequently used to evaluate for discoligamentous injury. Of the 223 such patients presenting to our level 1 trauma center over a 10year period, only 11 had acute soft tissue injuries identified on MRI that were not initially identified on CT examination. None of these injuries met criteria for classification of an unstable cervical spine injury, as defined by Denis's 3-column theory. ${ }^{16,19}$ Furthermore, mechanism of injury was not correlated with MRI-identified instability. Of the patients with positive MRI findings, 9 were treated with either soft or rigid cervical collar, none were treated surgically, and no patients with followup data went on to develop radiographic or clinical instability of their cervical spine or require delayed surgical treatment. Assessment of time-to-MRI data showed that nearly 10.5 hours elapsed between the time MRI was obtained and an attending radiologist read was documented. Nearly 20 hours elapsed between the time an initial CT scan was obtained to the point a final MRI read from an attending radiologist was available, which correlates closely with these patients' median duration of hospitalization.

As few patients $(5 \%)$ had positive MRI findings and no patients had unstable injuries requiring surgical treatment, our results suggest that MRI does not change treatment for neurologically intact blunt trauma patients with persistent neck pain and negative cervical spine CT imaging. Rather, MRI appears to delay patient disposition, increase hospitalization time, and create an overall disruption in patient care. In our study, time elapsed between initial $\mathrm{CT}$ scan and attending radiologist MRI read was used as a surrogate to represent the

Table 2. Outcomes in patients with positive magnetic resonance imaging (MRI) findings.

\begin{tabular}{|c|c|c|c|c|c|}
\hline Age (y) & Gender & Mechanism & MRI Diagnosis & Treatment & Outcome/Follow-Up \\
\hline 62 & Male & Motor vehicle collision & Interspinous ligament sprain & Aspen collar & Patient self-discontinued collar \\
\hline 27 & Female & Yoga & Interspinous ligament sprain & Soft cervical collar & $\begin{array}{l}\text { Unrelated emergency department visit } 6 \text { mo } \\
\text { later; no collar or neck pain }\end{array}$ \\
\hline 25 & Female & Motor vehicle collision & Interspinous ligament sprain & Soft cervical collar & No follow-up \\
\hline 36 & Male & Fell down intoxicated & Interspinous ligament sprain & Aspen collar & No follow-up \\
\hline 18 & Female & Assault & Interspinous ligament sprain & Aspen collar & $\begin{array}{l}\text { Unrelated emergency department visit } 5 \text { mo } \\
\text { later; no collar or neck pain }\end{array}$ \\
\hline 91 & Female & Ground-level fall & Interspinous ligament sprain & Soft cervical collar & 2 wk clinic follow-up; no neck pain \\
\hline 37 & Female & Fell from height & Interspinous ligament sprain & Aspen collar & No follow-up \\
\hline 65 & Male & Auto versus pedestrian & Interspinous ligament sprain & Aspen collar & No follow-up \\
\hline 37 & Female & Motor vehicle collision & Interspinous ligament sprain & Collar cleared & No follow-up \\
\hline 33 & Female & Motor vehicle collision & Interspinous ligament sprain & Collar cleared & No follow-up \\
\hline 37 & Male & Auto versus pedestrian & Prevertebral edema & Soft cervical collar & Patient self-discontinued collar \\
\hline
\end{tabular}


Table 3. Timing of computed tomography (CT), magnetic resonance imaging (MRI), and hospitalization. ${ }^{a}$

\begin{tabular}{lrrr}
\hline Time Period & Median & $\begin{array}{c}\text { First } \\
\text { Quartile }\end{array}$ & $\begin{array}{c}\text { Third } \\
\text { Quartile }\end{array}$ \\
\hline CT study to MRI study & $7: 45$ & $3: 58$ & $20: 31$ \\
MRI study to radiologist read & $10: 27$ & $2: 56$ & $17: 25$ \\
CT study to MRI radiologist read & $19: 43$ & $11: 27$ & $41: 08$ \\
Total hospitalization & $18: 43$ & $12: 52$ & $109: 05$
\end{tabular}

${ }^{\mathrm{a}}$ All times reported as hours:minutes. Radiologist read is the final attending radiologist interpretation of imaging studies.

total time required for a complete evaluation of a blunt trauma cervical spine injury within our patient population. The attending radiologist's interpretation of MRI results often impacts ED disposition decisions. In this case, an average of 10.5 hours was required for final disposition after an MRI was obtained. This is in addition to the almost 8 hours of time required to obtain an MRI after the initial CT is completed. As such, the time required to obtain MRI results in this patient population leads to a delay of care that can contribute to ED crowding without providing any treatment benefit, measured to be greater than 19 hours in the average patient work-up.

It should be emphasized that this delay in care is not benign. First, our results show that these patients may be undergoing unnecessary immobilization of the cervical spine with a rigid orthosis until an unstable injury is ruled out with MRI. In a study published in the Journal of Emergency Medicine, Schnabel et $\mathrm{al}^{20}$ performed a randomized controlled trial studying the effects of immediate cervical collar use and immobilization in patients with persistent neck pain due to stable whiplash injuries compared with early collar clearance and mobilization exercises. They found that the patients whose cervical spines were immobilized had significantly greater neck pain, headaches, and shoulder pain 6 weeks out from their injuries as well as higher disability scores and worse visual analog pain scores.

Second, our study demonstrates that nearly 20 hours of ED time or inpatient hospitalization is required when an MRI is utilized for cervical spine clearance in this patient population. In a study out of Temple University Hospital in 2017, Schreyer and Martin $^{21}$ calculated the cost of boarding patients in the ED to be $\$ 58.20$ per patient bedhour due to staffing and a higher level of care requirements. For our patient population, this translates to nearly $\$ 1100$ in direct cost to the hospital, per patient, for cervical collar clearance using MRI for the neurologically intact patients with negative $\mathrm{CT}$ imaging. The cost to the patient is similarly burdensome and unnecessary. The raw CPT billing charge in 2015 at our institution was \$456 per cervical spine MRI examination. This does not encompass the physician and radiology fees incurred by the patient, which further increases costs. In 2018, $\mathrm{Wu}$ et $\mathrm{al}^{14}$ performed a Markov model-based economic analysis for cost efficacy measured in quality-adjusted life years and determined that an MRI had no utility as a cost-effective imaging modality in neurologically intact patients with negative cervical spine $\mathrm{CT}$ imaging.

Prior studies evaluating the utility of MRI among neurologically intact blunt trauma patients with negative CT imaging have yielded mixed results. Ackland et $\mathrm{al}^{8}$ studied 178 patients with persistent midline tenderness and negative $\mathrm{CT}$ and found that $46 \%$ had injuries identified only on MRI. However, the presence of an MRI-detected injury was not associated with clinical outcomes at 12 months. Ackland et $\mathrm{al}^{7}$ separately reported that 5 patients in their population of 178 patients with positive MRI findings underwent surgical stabilization; however, their definition of unstable injury requiring surgery has been called into question. ${ }^{4}$ Difficulty in defining instability reflects the important finding that MRI has a high false-positive rate. In fact, many patients with MRI findings suggestive of ligamentous injury are found to have no injury at the time of surgery. ${ }^{2}$ Conversely, Raza et $\mathrm{al}^{12}$ found that CT had a $99.7 \%$ negative predictive value for ruling out cervical spine injury in obtunded blunt trauma patients, negating the need for further work-up with MRI. The most comprehensive analysis to date of MRI in neurologically intact blunt trauma patients with negative cervical spine imaging includes a metaanalysis by Malhotra et $\mathrm{al}^{4}$ with a total population of 5286 patients from 23 studies. Our results are consistent with those data, which demonstrated a very low overall rate of MRI-identified unstable injury. However, these authors note that there was significant heterogeneity among the included studies and that further studies, such as the present study, are necessary to further evaluate the utility of MRI in evaluating blunt cervical spine injury. To our knowledge, ours is also the first study to evaluate delay of care from time required to obtain MRI results.

Our results have important clinical implications. First, MRI did not identify additional injuries requiring surgical stabilization, suggesting that $\mathrm{CT}$ 
alone is appropriate for cervical spine clearance in blunt trauma patients without objective neurological injury. Second, our results further demonstrate that eliminating MRI from the work-up of persistent cervical spine pain in this patient population will help to reduce significant delay in patient care. Reducing the implementation of MRI to assess for cervical spine injury may decrease ED stay by up to 20 hours. In turn, this may shorten cervical spine immobilization times, thus directly improving shortterm patient outcomes ${ }^{20}$ as well as reduce ED overcrowding to drive down cost to the hospital and the patient.

Our study is not without its limitations. This is a retrospective study performed at a single center, reducing the study's overall generalizability. Additional limitations include inadequate availability of follow-up data for patients with MRI-identified injuries, which limits our ability to assess whether patients went on to develop instability over the long term. Strengths of the study include a large cohort of sequential patients, robust data available from our institution's electronic medical record, and being the first study to report on time required to obtain MRI.

In summary, we report on a large cohort of blunt trauma patients with negative CT scans who underwent MRI for assessment of missed, unstable injury in the setting of persistent cervical spine pain. We found that MRI has limited utility in detecting additional injuries among this population regardless of injury mechanism. Ours is also the first study to report on time required to obtain MRI and resultant delays in care for blunt trauma patients with persistent neck pain and negative CT.

\section{REFERENCES}

1. Gonzalez RP, Cummings GR, Phelan HA, Bosarge PL, Rodning CB. Clinical examination in complement with computed tomography scan: an effective method for identification of cervical spine injury. J Trauma. 2009;67(6):1297-1304.

2. Jo AS, Wilseck Z, Manganaro MS, Ibrahim M. Essentials of spine trauma imaging: radiographs, CT, and MRI. Semin Ultrasound CT MR. 2018;39(6):532-550.

3. Daffner RH, Hackney DB. ACR Appropriateness Criteria on suspected spine trauma. $J$ Am Coll Radiol. 2007;4(11):762-775.

4. Malhotra A, Wu X, Kalra VB, et al. Utility of MRI for cervical spine clearance after blunt traumatic injury: a metaanalysis. Eur Radiol. 2017;27(3):1148-1160.

5. Young AJ, Wolfe L, Tinkoff G, Duane TM. Assessing incidence and risk factors of cervical spine injury in blunt trauma patients using the National Trauma Data Bank. Am Surg. 2015;81(9):879-883.

6. Dunham CM, Brocker BP, Collier BD, Gemmel DJ. Risks associated with magnetic resonance imaging and cervical collar in comatose, blunt trauma patients with negative comprehensive cervical spine computed tomography and no apparent spinal deficit. Crit Care. 2008;12(4):R89. doi: 10.1186/ cc6957

7. Ackland HM, Cameron PA, Varma DK, et al. Cervical spine magnetic resonance imaging in alert, neurologically intact trauma patients with persistent midline tenderness and negative computed tomography results. Ann Emerg Med. 2011;58(6):521-530.

8. Ackland HM, Cameron PA, Wolfe R, et al. Outcomes at 12 months after early magnetic resonance imaging in acute trauma patients with persistent midline cervical tenderness and negative computed tomography. Spine (Phila Pa 1976). 2013;38(13):1068-1081.

9. Ackland HM, Wolfe R, Cameron PA, et al. Health resource utilisation costs in acute patients with persistent midline cervical tenderness following road trauma. Injury. 2012;43(11):1908-1916.

10. Mavros MN, Kaafarani HM, Mejaddam AY, et al. Additional imaging in alert trauma patients with cervical spine tenderness and a negative computed tomographic scan: is it needed? World J Surg. 2015;39(11):2685-2690.

11. Murphy JM, Park P, Patel RD. Cost-effectiveness of MRI to assess for posttraumatic ligamentous cervical spine injury. Orthopedics. 2014;37(2):e148-e152.

12. Raza M, Elkhodair S, Zaheer A, Yousaf S. Safe cervical spine clearance in adult obtunded blunt trauma patients on the basis of a normal multidetector CT scan - a meta-analysis and cohort study. Injury. 2013;44(11):1589-1595.

13. Schuster R, Waxman K, Sanchez B, et al. Magnetic resonance imaging is not needed to clear cervical spines in blunt trauma patients with normal computed tomographic results and no motor deficits. Arch Surg. 2005;140(8):762-766.

14. Wu X, Malhotra A, Geng B, et al. Cost-effectiveness of magnetic resonance imaging in cervical spine clearance of neurologically intact patients with blunt trauma. Ann Emerg Med. 2018;71(1):64-73.

15. Maung AA, Johnson DC, Barre K, et al. Cervical spine MRI in patients with negative CT: a prospective, multicenter study of the Research Consortium of New England Centers for Trauma (ReCONECT). J Trauma Acute Care Surg. 2017;82(2):263-269.

16. Denis F. Spinal instability as defined by the three-column spine concept in acute spinal trauma. Clin Orthop Relat Res. 1984;1984(189):65-76.

17. Haider AH, Chang DC, Haut ER, Cornwell EE III, Efron DT. Mechanism of injury predicts patient mortality and impairment after blunt trauma. J Surg Res. 2009;153(1):138142.

18. Sathiyakumar V, Thakore RV, Greenberg SE, et al. Adverse events in orthopaedics: is trauma more risky? an analysis of the NSQIP data. J Orthop Trauma. 2015;29(7):337341.

19. Denis F. The three column spine and its significance in the classification of acute thoracolumbar spinal injuries. Spine (Phila Pa 1976). 1983;8(8):817-831.

20. Schnabel M, Ferrari R, Vassiliou T, Kaluza G. 
Randomised, controlled outcome study of active mobilisation compared with collar therapy for whiplash injury. Emerg Med J. 2004;21(3):306-310.

21. Schreyer KE, Martin R. The economics of an admissions holding unit. West J Emerg Med. 2017;18(4):553-558.

Disclosures and COI: The authors received no funding for this study. Dr. Klineberg has worked as a consultant for DePuy Synthes Spine, Stryker, and Medicrea. He has also received honoraria and a fellowship grant for University of California, Davis, from AOSpine. Dr. Javidan has received honoraria and a fellowship grant for University of California, Davis from AOSpine. He has worked as a consultant for NuVasive, Alphatec, and Stryker, holds stocks in Alphatec, and has served as a consultant for Medicrea. Drs. Fennessy, Wick, Scott, and,
Roberto have no potential or perceived conflicts of interest.

Corresponding Author: Joseph Wick, MD, Department of Orthopaedic Spine Surgery, University of California, Davis, Medical Center, 4826 Y Street, Suite 3800, Sacramento, CA 95817. Phone: (507) 421-8698; Email: jbwick@ucdavis.edu.

Published 22 January 2021

This manuscript is generously published free of charge by ISASS, the International Society for the Advancement of Spine Surgery. Copyright (C) 2020 ISASS. To see more or order reprints or permissions, see http://ijssurgery.com. 